\title{
Body Mass Index can Similarly Predict the Presence of Multiple Cardiovascular Risk Factors in Middle-aged Japanese Subjects as Waist Circumference
}

\author{
Hiroki Satoh $^{1,2}$, Reiko Kishi ${ }^{2}$ and Hiroyuki Tsutsui ${ }^{1}$
}

\begin{abstract}
Objective Adiposity is closely associated with the clustering of metabolic risk factors such as high blood pressure, dyslipidemia, and glucose intolerance. Waist circumference and body mass index (BMI) are the established markers of abdominal adiposity and general adiposity, respectively. However, it has not been examined whether these two markers can detect the clustering of metabolic risk factors in Japanese subjects.

Methods and Results We studied 5,796 Japanese middle-aged subjects aged 40-60 years (4,344 males and 1,452 females). Metabolic risk factors including high blood pressure, dyslipidemia, and glucose intolerance were identified according to the diagnostic criteria for metabolic syndrome in Japan. The number of metabolic risk factors was significantly associated with the BMI values in both male and female subjects. The prevalence of subjects with multiple (two or more) metabolic risk factors was $29.4 \%$ and $7.6 \%$ in males and females, respectively. According to receiver operating characteristic (ROC) analysis, the area under curve values of BMI and waist circumference did not differ in male (0.658 vs. $0.671, \mathrm{p}=\mathrm{n}$.s. $)$ and female $(0.776$ vs. $0.790, p=n . s$.$) subjects, indicating that the waist circumference as well as the BMI could be useful in detect-$ ing the occurrence of multiple metabolic risk factors. The appropriate cut-off values of BMI to predict the presence of multiple metabolic risk factors were 24.7 and $23.4 \mathrm{~kg} / \mathrm{m}^{2}$ in males and females, respectively. The sensitivity and specificity using these cut-off values were 58 and $65 \%$ in males and 65 and $77 \%$ in females, respectively.

Conclusion The BMI values can similarly predict the presence of multiple metabolic risk factors just as the waist circumference in Japanese middle-aged subjects.
\end{abstract}

Key words: body mass index, waist circumference, risk factor, epidemiology, middle-aged Japanese subjects

(Inter Med 49: 977-982, 2010)

(DOI: 10.2169/internalmedicine.49.3006)

\section{Introduction}

Adiposity is associated with the accumulation of metabolic risk factors such as high blood pressure, dyslipidemia, and glucose intolerance, which may increase the risk of cardiovascular morbidity and mortality (1-6). Adiposity can be generally assessed by the measurement of waist circumference and body mass index (BMI). Previous studies have demonstrated that the waist circumference is the superior marker to predict cardiovascular risk factors than
BMI (7-10). In contrast, other studies have reported that both BMI and waist circumference values can equally identify cardiovascular risk factors (11-13). The American Diabetes Association has stated that it is unclear whether waist circumference can predict cardiovascular risk factors beyond BMI (14). These results indicate that the validity of these two markers to detect cardiovascular risk factors is a controversial issue. This is considered to be of clinical importance particularly because Asian populations have a higher proportion of percentage of body fat than Caucasians for a given BMI (15). Nevertheless, the relationship between adiposity

${ }^{1}$ Department of Cardiovascular Medicine, Hokkaido University Graduate School of Medicine, Sapporo and ${ }^{2}$ Department of Public Health, Hokkaido University Graduate School of Medicine, Sapporo

Received for publication October 12, 2009; Accepted for publication February 1, 2010

Correspondence to Dr. Hiroki Satoh, h-satoh@imb.me-h.ne.jp 
markers such as BMI and waist circumference and cardiovascular risk factors has not been fully established in Japanese populations. Moreover, the association between these two markers and the clustering of cardiovascular risk factors has not been studied.

In this study, we compared the ability of waist circumference and BMI values to predict the occurrence and clustering of cardiovascular risk factors in middle-aged Japanese subjects.

\section{Methods}

\section{Study subjects}

The present study included 6,056 Japanese subjects who were employed in food and telephone service company in Hokkaido, aged from 40 to 60 years old [50 \pm 8 years, mean \pm standard deviation (SD)], and had an annual health check-up during the period between April 2008 and March 2009. A total of 260 subjects (199 males and 61 females) were excluded due to prior coronary artery disease (CAD) or stroke. Thus, a total of 5,796 subjects remained in the present analysis. The study protocol was approved at two study companies and informed consent was obtained from all subjects.

\section{Data collection}

Blood samples were obtained from antecubital vein in the morning after overnight fasting and serum samples were separated after centrifugation. HDL-C was measured after precipitation of apo B-containing lipoproteins with a commercial reagent containing phosphotungstate and magnesium chloride (Daiichi Pure Chemicals, Tokyo, Japan). Triglyceride was measured enzymatically (Kyowa Medex, Tokyo, Japan). Fasting plasma glucose was enzymatically determined by the hexokinase method (Shino-Test, Tokyo, Japan). Blood pressure was measured by a trained nurse using a standard mercury sphygmomanometer with the study subjects in the sitting position after at least a 5-minute rest. Body weight, height, and waist circumference were measured in the morning in the fasting state. Waist circumference was measured around the abdomen at the level of the navel at the late expiratory phase by using a tape measure. BMI was calculated as body weight $(\mathrm{kg})$ divided by squared height $\left(\mathrm{m}^{2}\right)$.

Metabolic risk factors were diagnosed based on the new definition released by the Japanese Committee for the Diagnostic Criteria of Metabolic Syndrome in April 2005 (16); 1) high blood pressure; systolic blood pressure $\geq 130$ $\mathrm{mmHg}$ and/or diastolic blood pressure $\geq 85 \mathrm{mmHg}$, 2) dyslipidemia; triglyceride $\geq 150 \mathrm{mg} / \mathrm{dl}$ and/or HDL-C $<40$ $\mathrm{mg} / \mathrm{dL}$, and 3) glucose intolerance; fasting plasma glucose $\geq 110 \mathrm{mg} / \mathrm{dl}$. Each factor was considered to be present when the subject was under medication. Two or more risk factors were defined as "multiple" risk factors.
Table 1. Clinical Characteristics of the Study Subjects

\begin{tabular}{lcc}
\hline & $\begin{array}{c}\text { Male } \\
(\mathrm{n}=4,344)\end{array}$ & $\begin{array}{c}\text { Female } \\
(\mathrm{n}=1,452)\end{array}$ \\
\hline Age (years) & $52 \pm 8$ & $50 \pm 7^{*}$ \\
Waist circumference $(\mathrm{cm})$ & $85 \pm 9$ & $78 \pm 9^{*}$ \\
Body mass index $\left(\mathrm{kg} / \mathrm{m}^{2}\right)$ & $23.9 \pm 3.4$ & $21.9 \pm 3.5^{*}$ \\
Systolic blood pressure (mmHg) & $126 \pm 19$ & $118 \pm 18^{*}$ \\
Diastolic blood pressure (mmHg) & $79 \pm 12$ & $71 \pm 12^{*}$ \\
Triglyceride (mg/dL) & $112(81-156)$ & $73(53-101)^{*}$ \\
HDL-cholesterol (mg/dL) & $55 \pm 14$ & $70 \pm 16^{*}$ \\
Fasting plasma glucose (mg/dL) & $100 \pm 28$ & $90 \pm 15^{*}$ \\
Medical history & & $8.4^{*}$ \\
Hypertension $(\%)$ & 16.8 & $1.2^{*}$ \\
Diabetes mellitus $(\%)$ & 6.0 & 2.1 \\
Dyslipidemia $(\%)$ & 1.8 & \\
\hline
\end{tabular}

Values are presented as mean $\pm \mathrm{SD}$, median (interquatile range) for skewed variables,

or percentage.

HDL; high-density lipoprotein cholesterol.

$* \mathrm{p}<0.05$ vs. male.

\section{Statistical analysis}

All analyses were performed separately according to gender. The clinical and biochemical data of the study subjects were expressed as means $\pm \mathrm{SD}$, a median (and interquartile range) for variables with a skewed distribution, and percentages. The differences between two groups were examined by the Student unpaired t test for variables distributed normally, or by the Wilcoxon rank-sum test for triglyceride, and by the $\chi^{2}$-test for the categorical variables. A one-way analysis of variance (ANOVA) was used to calculate the differences in continuous variables among the multiple groups. Receiver operating characteristic curve (ROC) analysis was used to determine the appropriate waist circumference according to male and female. We calculated the maximal sensitivity and specificity between waist circumference and multiple risk factors. According to the ROC curve, the appropriate point was defined as the closest point on the ROC curves to the point at 1-specificity of 0 and sensitivity of $100 \%$. A p value of less than 0.05 was considered to indicate statistical significance. All statistical analyses were performed by using the Statistical Package for Social Science (version 11.0).

\section{Results}

Table 1 shows the clinical characteristics of the study subjects. Male subjects were older and had greater waist circumference, BMI, systolic and diastolic blood pressure, triglyceride, fasting plasma glucose, and lower high density lipoprotein cholesterol than female subjects. The prevalence of medical history for hypertension and diabetes mellitus was significantly higher in males than in females.

Figure 1 shows the relationship between BMI values and the numbers of metabolic risk factors among the study subjects. The BMI values were significantly greater according to the increase in the numbers of metabolic risk factors in 

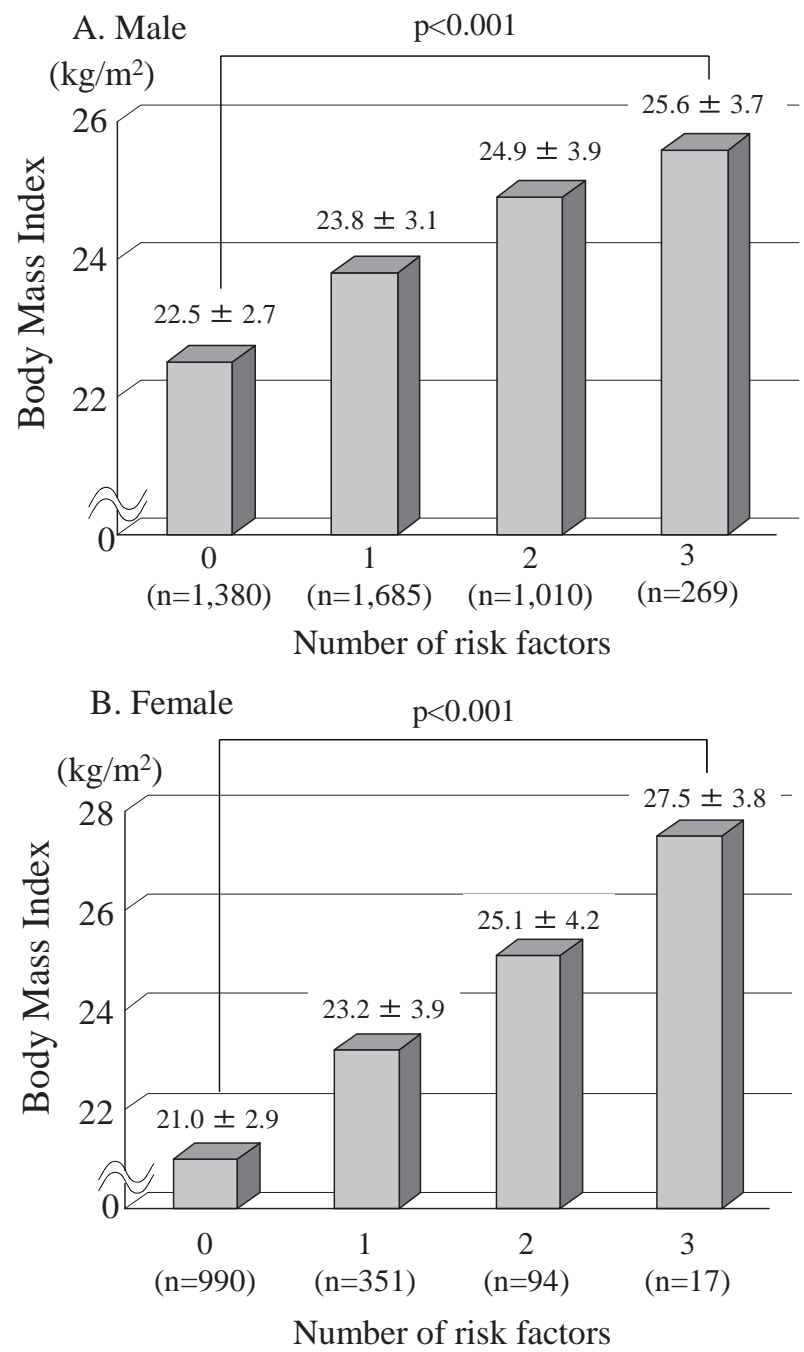

Figure 1. Body mass index values according to the numbers of metabolic risk factors in male (A) and female (B) subjects.

both males and females. The BMI values of the study subjects who had multiple risk factors were $25.0 \pm 3.5$ and 25.2 $\pm 4.2 \mathrm{~kg} / \mathrm{m}^{2}$ in males and females, respectively.

Figure 2 shows the relationship between waist circumference values and the numbers of metabolic risk factors among the study subjects. The waist circumference values were significantly greater according to the increase in the numbers of metabolic risk factors in both male and female subjects. The waist circumference values of the study subjects who had multiple risk factors were $89 \pm 6$ and $88 \pm 9$ $\mathrm{cm}$ in males and females, respectively.

Figure 3 shows the ROC curve to determine the appropriate BMI values for detecting the presence of multiple risk factors in males and females. In male subjects with the cutoff value of $24.7 \mathrm{~kg} / \mathrm{m}^{2}$, the sensitivity and specificity were $58 \%$ and $65 \%$, respectively, which were found to be the maximal values. In female subjects with the cut-off value of $23.4 \mathrm{~kg} / \mathrm{m}^{2}$, the sensitivity and specificity were $65 \%$ and $77 \%$, respectively, which were the maximal values. The sensitivity became as low as $47 \%$ and specificity was $88 \%$, when the cut-off value of $25 \mathrm{~kg} / \mathrm{m}^{2}$ was used in female sub-

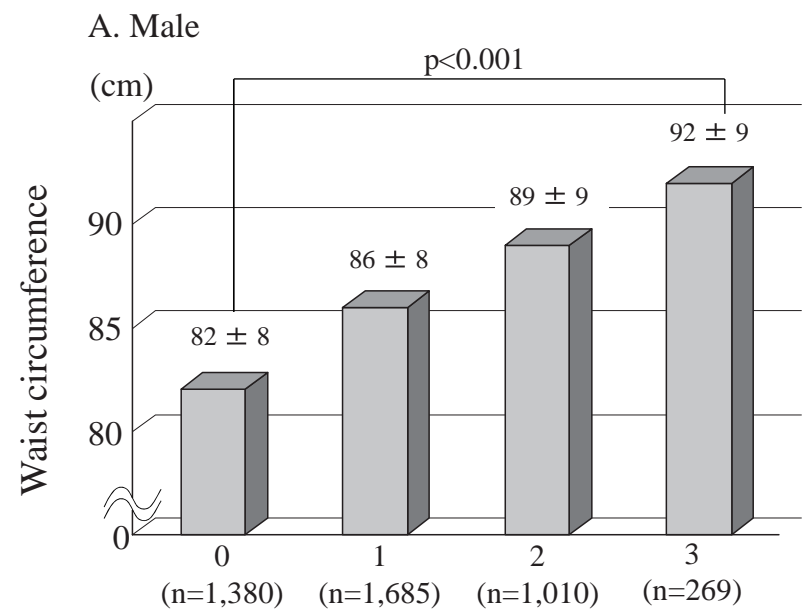

Number of risk factors

B. Female

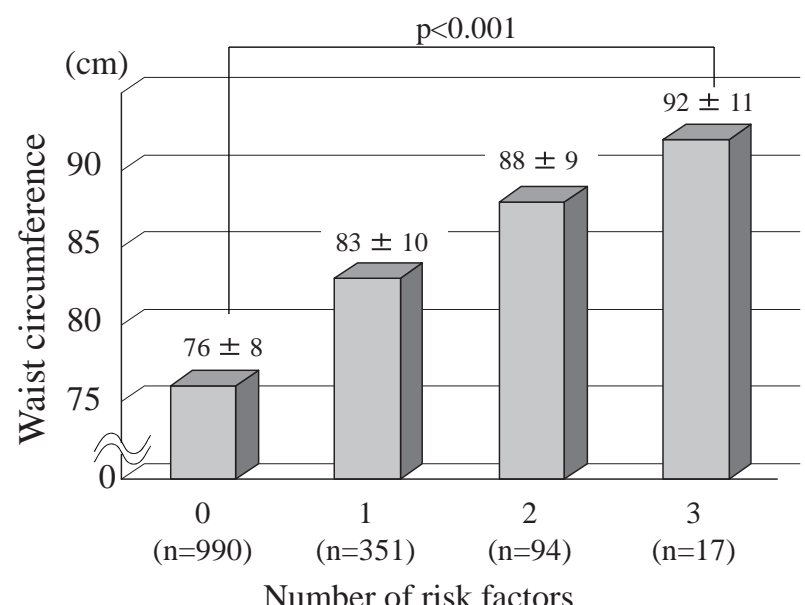

Figure 2. Waist circumference values according to the numbers of metabolic risk factors in male (A) and female (B) subjects.

jects.

Figure 4 shows the ROC curve to determine the appropriate waist circumference values for detecting the presence of multiple risk factors in males and females. In male subjects with the cut-off values of $86 \mathrm{~cm}$, the sensitivity and specificity were $62 \%$ and $64 \%$, respectively, which were found to be the maximal values. In female subjects with the cut-off value of $83 \mathrm{~cm}$, the sensitivity and specificity were $71 \%$ and $75 \%$, respectively, which were the maximal values. The sensitivity became as low as $47 \%$ and specificity was $88 \%$ when the waist circumference cut-off value of $90 \mathrm{~cm}$ was used in female subjects.

Table 2 shows the area under curve (AUC) values of waist circumference and BMI by using ROC analysis to detect multiple risk factors in both genders. AUC values did not differ between waist circumference and BMI in either males or females.

\section{Discussion}

The present study demonstrated that both waist circumfer- 


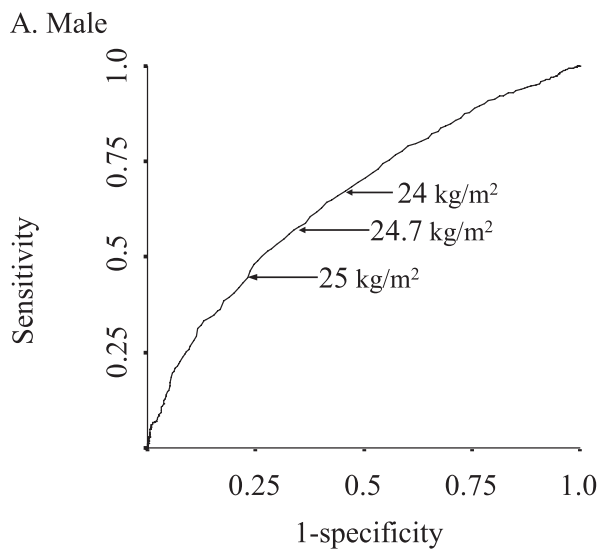

B. Female

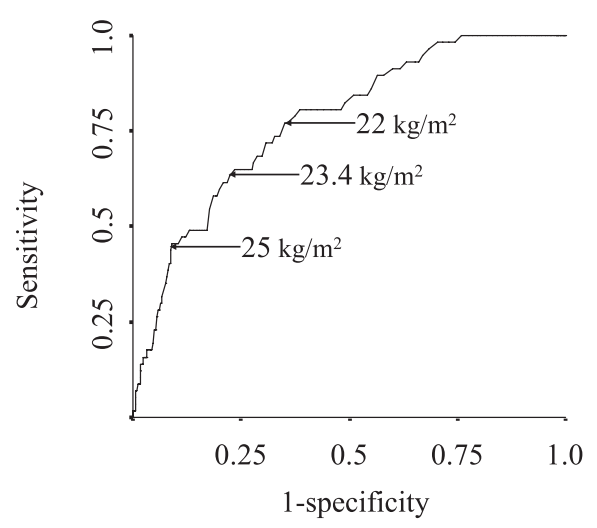

Figure 3. The ROC (receiver operating characteristic) curve for body mass index to detect multiple metabolic risk factors in male (A) and female (B) subjects. AUC: area under curve

ence and BMI values were equally useful markers to identify the presence of multiple cardiovascular risk factors in middle-aged Japanese subjects. The cut off values of waist circumference to predict multiple cardiovascular metabolic risk factors were $86 \mathrm{~cm}$ and $83 \mathrm{~cm}$ in males and females, respectively. Those of BMI were $24.7 \mathrm{~kg} / \mathrm{m}^{2}$ and $23.4 \mathrm{~kg} / \mathrm{m}^{2}$ in males and females, respectively.

General and abdominal adiposity were closely associated with the risk of death and the occurrence of cardiovascular disease $(17,18)$. Insulin resistance due to adiposity is the pathophysiological basis for the presence of cardiovascular metabolic risk factors such as high blood pressure, dyslipidemia, and glucose intolerance (19). Clustering of these risk factors is now recognized as an emerging risk for coronary artery disease $(20,21)$. The adiposity was generally identified by the measurement of waist circumference and BMI. The World Health Organization and the National Institutes of Health provided guidelines for classifying body weight status based on BMI and demonstrated a close relation between BMI and cardiovascular risk factors $(22,23)$. Recently, waist circumference has been widely used as a surrogate marker of abdominal adiposity, because waist circumference is correlated with abdominal fat mass and it is more associated with cardiovascular risk factors than BMI (7-10). However, the validity of these two markers to detect the presence of cardiovascular risk factors has been controver-

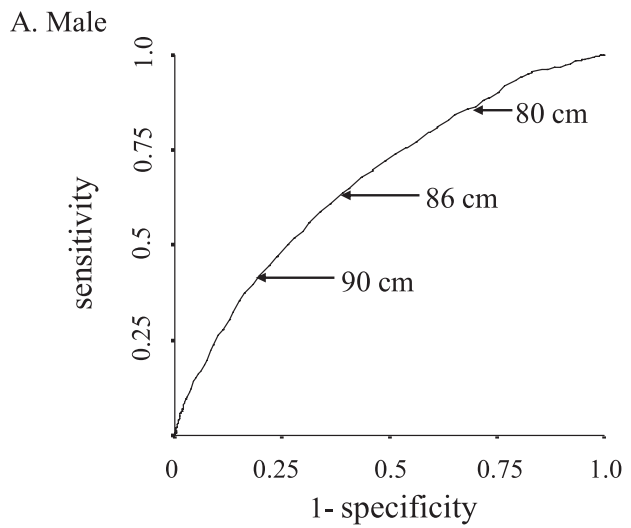

B. Female

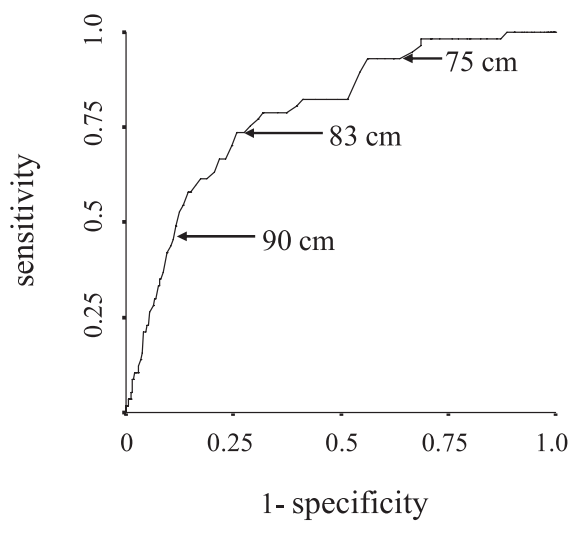

Figure 4. The ROC (receiver operating characteristic) curve for the waist circumference to detect multiple metabolic risk factors in male (A) and female (B) subjects. AUC: area under curve

Table 2. Area under Curves of Waist Circumference and Body Mass Index for Multiple Risk Factors

\begin{tabular}{cccc}
\hline & Body mass index & Waist circumference & p-value \\
\hline Male & & & \\
AUC & 0.658 & 0.671 & n.s. \\
$95 \%$ CI & $0.636-0.692$ & $0.650-0.692$ & \\
Female & & & \\
AUC & 0.776 & 0.790 & n.s. \\
$95 \%$ CI & $0.719-0.834$ & $0.734-0.847$ & \\
\hline
\end{tabular}

AUC, area under curves; CI, confidence interval.

sial among the reports. Shen et al reported that both waist circumference and BMI were closely correlated with total body adipose tissue mass, but waist circumference was a better predictor to detect visceral obesity than BMI in Caucasians (24). Similarly, Klein et al demonstrated that adipose fat mass was more associated with waist circumference than BMI (14). However, Lear et al reported that Asians had a higher percentage of body fat than Caucasians despite a given BMI (15). These results suggested that the distribution of body fat mass in Asians differed from Caucasians and 
BMI might be superior to detect visceral obesity in Asians than in Caucasians. The present study demonstrated that both BMI and waist circumference could equally predict the presence of multiple cardiovascular risk factors in Japanese population.

The World Health Organization Western Pacific Region and others proposed the definition of obesity as BMI $\geq 25$ $\mathrm{kg} / \mathrm{m}^{2}$ on the Asia-Pacific region (25). Ishikawa-Takata et al demonstrated the degrees of BMI associated with cardiovascular risk factors were lower than those in Caucasians (26). Thus, the appropriate cut-off BMI values to detect the presence of multiple cardiovascular risk factors may be lower than $25 \mathrm{~kg} / \mathrm{m}^{2}$. The present study has demonstrated that this is the case.

Specific health examination has been newly started in Japan, in which the cut-off values of waist circumference were $85 \mathrm{~cm}$ in males and $90 \mathrm{~cm}$ in females $(16,27)$. However, Miyawaki et al analyzed data from 3,574 employees of a telephone company and their family members (2,947 males and 627 females) obtained from health examinations and demonstrated that the appropriate cut-off waist circumference values were $86 \mathrm{~cm}$ for males and $77 \mathrm{~cm}$ for females to detect multiple risk components by using Japanese criteria based on their visceral-fat area cut-off levels of $100 \mathrm{~cm}^{2}$ in males and $65 \mathrm{~cm}^{2}$ in females (28), in which the appropriate cut-off values of waist circumference were lower than those in specific health examination. Our results also confirmed these previous findings.

Baik demonstrated optimal waist circumference values of $84-86 \mathrm{~cm}$ for men and of $78-80 \mathrm{~cm}$ for women to detect multiple cardiovascular risk factors in Korean populations (29). Takahashi et al indicated that cut-off values to detect multiple cardiovascular risk factors were $85 \mathrm{~cm}$ (men) and $80 \mathrm{~cm}$ (women) in waist circumference and $24 \mathrm{~kg} / \mathrm{m}^{2}$ (men) and $23 \mathrm{~kg} / \mathrm{m}^{2}$ (women) in BMI in Japanese popula- tions and the combination of both waist circumference and BMI was more useful than using only one of these parameters (30). Both sensitivity and specificity increased by using the combination of these two markers; however, the equivalent interaction as a trade off was generally present in evaluating a screening test and the statistical accuracy might be limited in using this strategy. The appropriate cut-off values of waist circumference and BMI in our study confirmed the results of these previous studies. However, these studies could not investigate the comparison and superiority between waist circumference and BMI as a screening tool to identify the presence of multiple cardiovascular risk factors, which differed from the present study.

There are several limitations that should be acknowledged in this study. First, our study subjects ranged from 40 to 60 years old. Therefore, we have to be cautious in extending the present results to the general population. Second, even though visceral obesity is considered to be associated with $\mathrm{CAD}$, we did not obtain the outcome data including the occurrence of CAD in this study. Further studies are needed to evaluate the association between BMI or waist circumference and future occurrence of cardiovascular events to define the appropriate cut-off values of waist circumference in the Japanese population.

\section{Conclusions}

The present study concluded that both BMI and waist circumference values were associated with cardiovascular risk factors, and BMI values can equally predict multiple cardiovascular risk factors as waist circumference in middle-aged Japanese subjects. It is one of the important instructions for health promotion strategy to maintain appropriate BMI and waist circumference values by lifestyle modification including diet and exercise.

\section{References}

1. Oda E, Kawai R. Age- and gender-related differences in correlations between abdominal obesity and obesity-related metabolic risk factors in Japanese. Intern Med 48: 497-502, 2009.

2. Whitlock G, Lewington $S$, Sherliker P, et al. Body-mass index and cause-specific mortality in 900000 adults: collaborative analyses of 57 prospective studies. Lancet 373: 1083-1096, 2009.

3. Sone H, Mizuno S, Fujii H, et al. Is the diagnosis of metabolic syndrome useful for predicting cardiovascular disease in asian diabetic patients? Analysis from the Japan Diabetes Complications Study. Diabetes Care 28: 1463-1471, 2005.

4. Lakka HM, Laaksonen DE, Lakka TA, et al. The metabolic syndrome and total and cardiovascular disease mortality in middleaged men. JAMA 288: 2709-2716, 2002.

5. Isomaa B, Almgren P, Tuomi T, et al. Cardiovascular morbidity and mortality associated with the metabolic syndrome. Diabetes Care 24: 683-689, 2001.

6. Grundy SM, Brewer HB Jr, Cleeman JI, Smith SC Jr, Lenfant C. Definition of metabolic syndrome: Report of the National Heart, Lung, and Blood Institute/American Heart Association conference on scientific issues related to definition. Circulation 109: 433-438, 2004.
7. Huang KC, Lee MS, Lee SD, et al. Obesity in the elderly and its relationship with cardiovascular risk factors in Taiwan. Obes Res 13: 170-178, 2005.

8. Misra A, Madhavan M, Vikram NK, Pandey RM, Dhingra V, Luthra K. Simple anthropometric measures identify fasting hyperinsulinemia and clustering of cardiovascular risk factors in Asian Indian adolescents. Metabolism 55: 1569-1573, 2006.

9. Thomas GN, Ho SY, Lam KS, Janus ED, Hedley AJ, Lam TH. Impact of obesity and body fat distribution on cardiovascular risk factors in Hong Kong Chinese. Obes Res 12: 1805-1813, 2004.

10. Zhu S, Heymsfield SB, Toyoshima H, Wang Z, Pietrobelli A, Heshka S. Race-ethnicity-specific waist circumference cutoffs for identifying cardiovascular disease risk factors. Am J Clin Nutr 81: 409-415, 2005.

11. Farin HM, Abbasi F, Reaven GM. Comparison of body mass index versus waist circumference with the metabolic changes that increase the risk of cardiovascular disease in insulin-resistant individuals. Am J Cardiol 98: 1053-1056, 2006.

12. Pankow JS, Jacobs DR Jr, Steinberger J, Moran A, Sinaiko AR. Insulin resistance and cardiovascular disease risk factors in children of parents with the insulin resistance (metabolic) syndrome. 
Diabetes Care 27: 775-780, 2004.

13. Reaven G. All obese individuals are not created equal: insulin resistance is the major determinant of cardiovascular disease in overweight/obese individuals. Diab Vasc Dis Res 2: 105-112, 2005.

14. Klein S, Allison DB, Heymsfield SB, et al. Waist circumference and cardiometabolic risk: a consensus statement from shaping America's health: Association for Weight Management and Obesity Prevention; NAASO, the Obesity Society; the American Society for Nutrition; and the American Diabetes Association. Diabetes Care 30: 1647-1652, 2007.

15. Lear SA, Humphries KH, Kohli S, Birmingham CL. The use of BMI and waist circumference as surrogates of body fat differs by ethnicity. Obesity (Silver Spring) 15: 2817-2824, 2007.

16. Matsuzawa Y. Metabolic syndrome-Definition and diagnostic criteria in Japan. J Jpn Soc Int Med 94: 188-203, 2005 (in Japanese).

17. Balkau B, Deanfield JE, Despres JP, et al. International Day for the Evaluation of Abdominal Obesity (IDEA): a study of waist circumference, cardiovascular disease, and diabetes mellitus in 168,000 primary care patients in 63 countries. Circulation 116: 1942-1951, 2007.

18. Pischon T, Boeing H, Hoffmann K, et al. General and abdominal adiposity and risk of death in Europe. N Engl J Med 359: 21052120, 2008.

19. Wajchenberg BL. Subcutaneous and visceral adipose tissue: their relation to the metabolic syndrome. Endocr Rev 21: 697-738, 2000.

20. Reaven G. The metabolic syndrome or the insulin resistance syndrome? Different names, different concepts, and different goals. Endocrinol Metab Clin North Am 33: 283-303, 2004.

21. Kashyap SR, Defronzo RA. The insulin resistance syndrome: physiological considerations. Diab Vasc Dis Res 4: 13-19, 2007.

22. Clinical Guidelines on the Identification, Evaluation, and Treatment of Overweight and Obesity in Adults--The Evidence Report. National Institutes of Health. Obes Res 6 (Suppl 2): 51S-209S, 1998.

23. World Health Organization: Obesity: Preventing and managing the global epidemic: Report of a WHO Consultation on Obesity. Geneva, World Health Organization 1998.

24. Shen W, Punyanitya M, Wang Z, et al. Total body skeletal muscle and adipose tissue volumes: estimation from a single abdominal cross-sectional image. J Appl Physiol 97: 2333-2338, 2004.

25. World Health Organization Western Pacific Region, International Association for the Study of Obesity, International Obesity Task Force. The Asian-Pacific Perspective: Redefining Obesity and its Treatment. World Health Organization Pacific Region, Geneva, 2000.

26. Ishikawa-Takata $\mathrm{K}$, Ohta $\mathrm{T}$, Moritaki $\mathrm{K}$, Gotou $\mathrm{T}$, Inoue $\mathrm{S}$. Obesity, weight change and risks for hypertension, diabetes and hypercholesterolemia in Japanese men. Eur J Clin Nutr 56: 601-607, 2002.

27. The Examination Committee of Criteria for 'Obesity Disease' in Japan, Japan Society for the Study Obesity. New criteria for 'obesity disease' in Japan. Circ J 66: 987-992, 2002.

28. Miyawaki T, Hirata M, Moriyama K, et al. Metabolic syndrome in Japanese diagnosed with visceral fat measurement by computed tomography. Proc Japan Acad 81: 471-479, 2005.

29. Baik I. Optimal cutoff points of waist circumference for the criteria of abdominal obesity: comparison with the criteria of the International Diabetes Federation. Circ J 73: 2068-2075, 2009.

30. Takahashi M, Shimomura K, Proks P, et al. A proposal of combined evaluation of waist circumference and BMI for the diagnosis of metabolic syndrome. Endocr J 56: 1079-1082, 2009.

(C) 2010 The Japanese Society of Internal Medicine http://www.naika.or.jp/imindex.html 